\title{
Characteristic of the Naming of Hotels in Tengchong Heshun Ancient Town
}

\author{
Meian Zhang \\ Baoshan University, Baoshan Yunnan, 678000, China
}

Keywords: Tengchong Heshun, Hotel naming, Characteristic

\begin{abstract}
Heshun ancient town in Tengchong, Yunnan is a famous home town of overseas Chinese and one of the top ten charming towns in China; furthermore, it is a well-known tourist attraction in China. There are nearly 200 hotels at local. This paper discusses the characteristic of the hotel naming in Heshun ancient town from the characteristic of lingual formation, the characteristic of content component and characteristic of cultural psychology in hotel naming for the purpose of exploring the language application in local hotel naming.
\end{abstract}

\section{The Basic Information of Heshun Ancient Town and Heshun Hotels}

Tengchong Heshun ancient town is a famous tourist attraction in China and it has a long cultural history. The ancient town is located at the west of Tengchong City, with a distance of four kilometers. It was built from Ming Dynasty. Heshun ancient town is a well-known hometown for overseas Chinese in Yu Nan province and also the key town in Ancient Tea Horse Road and the route that must be taken in southwestern Silk Road. Heshun ancient road is praised as the hometown of jadeite created by transportation of caravan and the window of Han culture mingling with south Asia and western culture. In the evaluation and selection event of Chinese charming towns held by CCTV in 2005, Heshun ancient town and its unique charm as well as the harmonious life of local 6000 residents shot to fame overnight and won the title of top ten charming towns in China and the only annual award. Because of Heshun ancient town's beautiful natural scenery, antique buildings and long and profound history and culture, it becomes a tourist attraction for tourists from China, even from the world. The development of tourist industry in Heshun ancient town inevitably brings the booming of hotels. Now there are 200 hotels of different sizes in Heshun ancient towns, mainly distributed in residents' houses in the ancient town and the majority of them is family hotels.

\section{The Characteristics of Heshun Ancient Town Hotel Naming}

The characteristic of lingual formation of hotel naming.

Syllable is neat and even, mainly in even number

There are around 200 hotels in Heshun ancient town. I analyze the names of 180 of the hotels. It is found that the majority of Hushun hotels have 4 syllables or 6 syllables, followed by 7 syllables and 5 syllables, with 3 syllables at the bottom, and there are zero hotel with 1 syllable or 2 syllables. The detailed research result is showed as in following chart:

\begin{tabular}{|c|c|c|c|c|c|c|}
\hline $\begin{array}{c}\text { total number } \\
\text { of hotels }\end{array}$ & $\begin{array}{c}3 \\
\text { syllables }\end{array}$ & 4 syllables & 5 syllables & 6 syllables & 7 syllables & Above 7 syllables \\
\hline 180 & 3 & 45 & 20 & 46 & 31 & 35 \\
\hline percentage & $1.7 \%$ & $25 \%$ & $11.1 \%$ & $25.6 \%$ & $17.2 \%$ & $19.4 \%$ \\
\hline example & $\begin{array}{c}\text { He Mei } \\
\text { Ju }\end{array}$ & $\begin{array}{c}\text { Er Cun } \\
\text { Ming Ju }\end{array}$ & $\begin{array}{c}\text { Yun Tian } \\
\text { Wai Ke Zhan }\end{array}$ & $\begin{array}{c}\text { Bian Cheng } \\
\text { Gu Shi Ke } \\
\text { Zhan }\end{array}$ & $\begin{array}{c}\text { He Shun Da Ma } \\
\text { Bang Ke Zhan }\end{array}$ & $\begin{array}{c}\text { He Shun San Cheng } \\
\text { Ha Ming Ju Ke } \\
\text { Zhan }\end{array}$ \\
\hline
\end{tabular}

It can be observed from the table that the majority of Heshun hotel naming has even number syllables, of which the syllable structure is neat and even. The names with above 7 syllable are also mainly in 8 syllables. China is a country with vast territory. Far back in ancient times, people had to struggle with wicked natural environment in order to survive. In challenging nature, people were fonder of diverse things. People always like to describe our country with vast territory and abundant resources. Thus, in comparison to other countries, Chinese are more like to the diversity of things. 
Such mentality is inevitably reflected in naming; therefore it is not hard to understand that the majority of Heshun hotel names are in 4 or above 4 syllables. Chinese also like to use "good things should be in pairs" to describe happy things in life. Happy events like wedding, moving to a better place and so on tend to be scheduled on even number dates, so the majority of hotels in Heshun ancient town chose even number syllable just reflects such mentality of Chinese. In these naming, 4 syllables and 6 syllables account for $50.6 \%$ of the total number because from the physiology perspective, the pronunciation of 4 syllables and 6 syllables are the easiest and it is convenient for memorizing and it is also very catchy. From an visual perspective, 4 syllables and 6 syllables are most natural and even, rich in rhythm. In the names of hotels in Heshun, above 7 syllables accounts for $19.4 \%$, while 3 syllables only accounts for $1.7 \%$ and there are zero hotel with 1 syllable or 2 syllables.

The alternation of tone pattern in intonation

In naming of 180 hotels, the names with oblique tones are only "Shan Shu Ke Zhan, Gu Zhen Yin Xiang, Jin Xiu Ke Zhan" and other three, but the names with all level tone are also only three, that is, "He Tian Ming Ju, Lan Xiang Ming Ju, An Xin Ming Ju." In Heshun ancient town, most hotels pay attention to name the hotels between oblique tones and level, so it reads with the sense of cadence and great sense of rhythm and musicality.

The usage of summation tone

"Summation tone, in ancient times was called 'tautology' or 'double words'. The proper application of reduplicated words, you can highlight the significance of the words, strengthen the image of the things, enhance the depiction of things and increase the sense of beauty in musice." (Huang Borong Liao Xudong, "Modern Chinese" Higher Education Press, 2011, p. 180) in Heshun hotel names, such as "Yi Yi Xiao Yuan," "Dong Dong Xiao Yua," "Shun Shun Ke Zhan", "Da Da Ke Zhan " and so on just adopted summation tone for naming.

Commonly used figure of speech

Exaggeration

"Deliberately exaggerating, enlarged, reduced or anticipatory description of objective people and things, this figure of speech is called exaggeration." (Huang Bo Rongliao Xudong, "Modern Chinese", Higher Education Press, 2011, p. 200) and Heshun hotels which used exaggeration for naming are "He Shun Yun Shang Shui Jing Ke Zhan", "Zi Yun Ju", "Yun Tian Wai Ke Zhan”, "Bai Sui Yuan Ke Zhan”. "He Shun Bai Nian Gu Zhai Ke Zhan”, "He Shun Wan Yuan Ke Zhan” "and so on. The names of these hotels are either related to sky, or related to time, making exaggerated description about things.

Quote

"Quoto", this figure of speech is rare in hotel naming, but there are a lot of hotels Heshun ancient town using it, such as "He Shun Nan Shan Ju Ke Zhan" quoted from Tao Yuanming's poem "Cai Ju Dong Li Xia, You Ran Xian Nan Shan"; "He Tang Yue Se Jing Ping Ke Zhan" quoted from Mr. Zhu Ziqing's "Lotus Pond"; "He Shun Wu Tuo Bang Guan Jing Ke Zhan" quoted from Europe founder of the early utopian socialist doctrines Thomas Moore’s (St. Thomas More) famous "Utopia"; "Ai Qing Gong Yu" directly quoted from the TV series "Ai Qing Gong Yu" as to be the hotel's name.

Analogy

Vesting people's thoughts and feelings in hotels gives a warm feeling such as "Yin He Ke Zhan", "Lian Yi Xian Ting Ke Zhan", etc. Because outside Heshun town is a lotus pond, Heshun people therefore particularly love lotus, so as to give the hotel the name Lotus.

Other such as yard "Yue Tai Xiao Yuan ","Ji Mu Xiao Wu" using the figurative speech; "Bei Ai Zheng Zhang Jia" using bailiff to replace hotel name, using the figure of speech of metonymy.

\section{The characteristic of content component of hotel naming.}

From the content of Heshun hotel naming, there is always such a feature: more than $80 \%$ of the hotel are named as "Ke Zhan". Ke Zhan is title of hotel in ancient times. Our modern people generally call the lodging places as hotels, but most hotels in Heshun ancient town are named as "Ke Zhan" that is in a harmony with the atmosphere of local antique brick tile and the cultural city with long history ,from which people will have a different feel once they stepped into the town. 
Specifically, the content of Heshun ancient town naming is very rich, it can be said as varied, dizzying. To sum up, there are the following naming modes:

Naming related to titles

This class of naming is plain, easy to remember, there are three naming modes:

Naming with family name:

He Shun Yi Jia Ke Zhan, Liu Jia Bie Yuan, Cun Jia Ke Zhan, Er Cun Ke Zhan( the hotel owners are two brothers with the family name of Cun) and so on

Naming with given name:

He Shun Hai Tao Ke Zhan, He Shun Rong Hui Ming Ju, Dong Dong Xiao Yuan, Lan Xiang Ming

Ju, He Shun You Peng Ming Ju, Hui Shun Ren Hui Ming Ju, Lin Li Ming Ju and so on

Naming with title

He Shun Yang Xiao Jie Ting Yuan, Teng Chong He Shun Wu E Ge Si Jia Ke Zhan and etc.

Naming related to place name

The naming with place names, on the one hand, is convenient to be found by tourists, on the other

hand, also convey the local place name information to tourists. There are mainly two modes as following;

Naming directly with the hotel address: :

No. 29 Gong Guan Xing Zhe Yi Zhan, Xiao Xiang No. 1, He Shun Xiao Jie Zi Ming Ju Ke Zhan, He Shun Gu Jin Lu Xiao Yuan, Ju Ren Xiang Ming Ju etc.

Naming with the unique tourist attraction in Heshun ancient town

He Shun Ma Que Guan Jing Ke Zhan, He Shun Shi Hua Men Ming Ju, Guan Jing Hu Ming Ju, He Shun Huang Guo Shu Ke Zhan and so on.

Naming related to people's certain wish and emotion

In Heshun hotel names, this class is the most, fully demonstrating people's yearning for the good things. There are mainly four naming modes:

Naming related to good wishes and blessings:

Fu Gu Lou, He Mei Ju, Liu Fu Ke Zhan, He Shun Fu Le Ju, Ru Yi Ke Zhan, Bai Sui Yuan Ke Zhan, He Shun Qian Xi Ming Ju, Fu Ren Ju Ke Zhan, Yong Ping Ke Zhan, Guo Sheng Ming Ju Ke Zhan, He Shun De Zi Zai Ju, Jiu Xiu Ke Zhan, Xing Fu Li Ke Zhan, Zi Rui Ju Ke Zhan, Ling Jun Ke

Zhan, He Shun Liang Ke Zhan, He Shun Tian Yuan Ju Ke Zhan and so on.

Naming related to nostalgia and hermit emotions

Bian Chen Gu Shi Ke Zhan, Xiao Xiang Shi Guang Ke Zhan, He Shun Da Ma Bang Ke Zhan, He Shun Bian Cheng Ji Yi Ke Zhan, San Ji Di Ke Zhan, Yin Shan Ju Ke Zhan, Lian Yi Xian Ting Ke Zhan, He Shun Cha Ma Yi Zhan, He Shun Bai Nian Ke Zhan, Qing Lian Ju Ke Zhan, San Shui Ming $\mathrm{Ju}$ and so on

Naming related to love

He Shun Ba Shu Yuan Ke Zhan, Ai Qing Gong Yu, He Shun TA TA Gu Shi Du Jia Lv Guan, Yin Hua Yuan Ke Zhan, He Shun Mei Gui Yuan Ke Zhan, He Shun Dian Tan Qing Ke Zhan and so on. Naming related to kindness, filial piety and Buddha

Shan Su Ke Zhan, He Shun Xiao Ren Ke Zhan, Xiao Shun Zhi Jia Gu Yi Ju Ke Zhan, He Shun

Chan Da Ke Zhan, He Shun Pu Ti Xi Ju and so on

Naming related to the features of hotels:

Mi Ni Xiao Wu, He Shun Xiao Ynag Lou Ke Zhan, Hua Da Men Gu Ming Ju, He Shun Jiu Jian

Fang Ke Zhan, Teng Bian Ke Zhan, He Shun Gu Dao Yang Sheng Ge and so on Naming related to TV drama names and poems and essays:

Ai Qing Gong Yu, He Shun Nan Shan Ju Ke Zhan, He Shun Wu Tuo Bang Guan Jing Ke Zhan,

He Shun He Tang Yue Se Jing Ping Ke Zhan

Others

Teng Chong He Shun Duo Cai Jing Lu Ke Zhan, No. 29 Gong Guan Zhi 6+1 Jing Ping Cha Yi Ke Zhan, He Shun Bo Li Heng Ke Zhan, He Shun Zui Yi Shi Guang Ying Yue Ke Zhang and so on. 


\section{The characteristic of cultural psychology in hotel naming.}

Language and culture are inextricably related. China's famous linguist Mr. Luo Changpei mentioned in "Language and Culture": the language on the one hand is an important part of culture, the record of cultural symbol systems; on the other hand, the respective cultural characteristics would inevitably influence and constrain language features and its using manner. Language is also an important medium for conveying information. The naming feature of Heshun ancient town hotels apparently is closely linked with the local people's cultural psychology. The hotels, as a social language, play an important role in communication between people, conveying much cultural psychological characteristics of local people to us.

Seeking for blessing

Everyone wants to have blessing. The desire for good luck, happiness and good health, peace and prosperity is common psychology of all people and is also the traditional culture of Chinese people. A large part of naming of Heshun ancient town hotels reflects the cultural psychological characteristics. "Liu Fu Ke Zhan, "Xing Fu Li Ke Zhan" conveys the owner's cultural psychology of desiring happiness to us."He Mei Ju", "Ru Yi Ke Zhan" express the owner's wish to live happily and as he likes it to be. "Guo Sheng Ming Ju Ke Zhan" shows the owner's good wish for the prosperity of the country. "Jiu Xiu Ke Zhan" let us feel the owner's love for beautiful rivers and mountains of the motherland. "Bai Sui Yuan Ke Zhn" He Shun Qian Xi Mingju " reflect people’s good wish to live longer. Among the near 200 hotels in Heshun ancient town, the hotel names that reflect the mentality to seek blessing account for quite a portion, which proves the psychological characteristics of people desiring for happiness and good life.

Seeking for profit

"May you make good fortune" is the greetings commonly used in our daily lives. Many stores also like to use its name implying to make a fortune. The naming of hotels in Heshun ancient town naturally also reflects the cultural psychology for seeking fortune. However, due to Heshun people long being influenced by Confucian scholarship from their childhood, their pursuit for profit is relatively indifferent, so this type of hotel name is quite small, There are only six hotels such as "Fu Gui Lou", "Hong Ying Ming Ju", "Wan Yuan Ke Zhan", "Jin Yue Lou Ke Zhan "' He Shun Gu Zheng Fu Kang Ke Zhan "and etc. As it can be seen from the hotel names, although it implies making a fortune and seeking for profit, yet its name is relatively obscure and it does not nakedly reflect the mentality for seeking profit and fortune.

Seeking for elegance

I visited the 180 hotels, .among which, quite a part of them having a delicate and elegant names, with a unique taste such as "Xiao Xiang Shi Guang Ke Zhan”, "He Shun Bian Chen Ji Yi Ke Zhan”, “Teng Chong He Shun Wei Yi Xing Ke Zhan”, "He Shun Qing Xuan Han She Jing Ping Ke Zhan”, "Bian Cheng Gu Shi Ke Zhan" and the similar names with bookish air, which reflects the profound cultural background of people in Heshun ancient town.

Seeking for rarity

Such hotels often have long names in order to attract people's attention, often with some strange names, which make people both feel fancy, but baffled such as "No.29 Gong Guan Zhi $6+1$ Zhi Jing Ping Ke Zhan ", "Teng Chong He Shun Duo Cai. Jing Lu Ke Zhan," "He Shun Ta Ta Gu Shi Du Jia Lv Guan" "Ge Zi De He Shun Shi Guang Ke Zhan" and so on. Although the names are eccentric, yet they also leave people a deep impression.

Seeking for real

The place names by title or place names reflect Heshun ancient town people's culture psychology for seeking real such as "He Shun Hai Tao Jia Ke Zhan ", "Liu Jia Bie Yuan", "Xiao Xiang No. 1," and so on named with real personal names or place names, which are plain to be understood and will never be forgot once tourists saw them.

Others

Some hotel names in Heshun ancient town also reflect the people's cultural and psychological characteristic for "seeking ancient, seeking vulgar, seeking large, seeking superior" and many other cultural psychological characteristics. However, regardless how hotel owners named the hotels, they 
are all based on the cultural psychology that people yearn for the good things and they wish their hotel names to be famous and impress people.

\section{Conclusion}

In short, the language is a product of society and reflects the stigma of the times, so are the hotel names, which reflect the common psychology that people yearn for good names. From the perspective of outside form, it need to be easy for memorizing, reading and looking; while from the perspective of content, it need to have beautiful meaning, implying good luck and happiness. Most of hotels in Tengchong Heshun ancient town have followed such rules, so in general the names of hotels meet with most people's aesthetic needs. Of course, there are a small part of the hotels with names too strange and letting people baffled. And part of the hotel names are the same, which is easy to get tourists confused. All of these are the place that needs to be improved.

\section{References}

[1] Huang Borong Liao Xudong “Modern Chinese”. Beijing. Higher Education Press. 2011.6

[2] Zhang Xiaoyu. The study over the store naming behavior from the perspective of linguistics. Chang Chun. Jilin University Master's Thesis .2006.5

[3] 2013.6 Shi Yanhua. Analysis on language culture food shop names in Tengchong .2013.6

[4] Gu Jinzi. Study on Chinese hotel name. Chang Chun. Jilin University Master's Thesis2014.6 